Review Article

\title{
A Narrative Review of Contemporary Global Perspectives into Epidemiology, Treatment, and Prognosis of Vertebrobasilar Strokes
}

\author{
Samwel Msigwa ${ }^{1^{*}}$ \\ ${ }^{1}$ Department of Neurology, The Clinical Medicine School of Yangtze University, the First Affiliated Hospital of Yangtze \\ University, Jingzhou, Hubei ,434023, China
}

*Corresponding author: Samwel Msigwa. Department of Neurology, The Clinical Medicine School of Yangtze University, the First Affiliated Hospital of Yangtze University, Jingzhou, Hubei ,434023, China. Email: neurovascular7@gmail.com

\begin{abstract}
Blood flow interruptions to the posterior cerebral circulation hallmark vertebrobasilar strokes (VBS), leading to mortality and significant disabilities, yet optimal therapy prevails unpublished. Recent epidemiological evidence indicates that VBS account for nearly 1/5 of all ischemic strokes globally, with acute basilar artery occlusion (BAO) contributing significant disabilities in nearly $1 / 3$ of the victims. The prevalence of VBS in Africa is close to $5 \%$, majorly in large intracranial vessels. Etiologically, Stenosis accounts for $20 \%$ of all VBS, while aneurysms face up to a 3\% rupture rate. Furthermore, intravenous alteplase is the gold standard medical therapy for the cases presenting within 3 to 4.5 hours post-baseline regarding management options. Nevertheless, there is no consensus for BAO beyond 4.5 hours post-onset. Stent retrievers are the first-line endovascular reperfusion therapy device proposed. However, an $18 \%$ risk of in-stent restenosis is a significant drawback. Comprehensive prognostic factors are addressed in this review. However, prospective, multicenter, controlled studies are needed to clarify the time window dilemmas facing posterior circulation strokes. This narrative review explores recent VBS epidemiology, management advances, and prognosis.
\end{abstract}

Rwanda J Med Health Sci 2021;4(3):418-429

Keywords: Stroke, Prognosis, Basilar Artery, Prevalence, Africa

\section{Introduction}

Large vessel occlusive strokes (LVOS), including vertebrobasilar strokes (VBS) globally account for nearly $14-25 \%$ of ischemic strokes (IS). The primary pathogenesis is centered on the build-up of atheroma at the sites of paramount turbulence, prevalently at the origin, vertebrobasilar (VB) juncture, and the mid-basilar artery. [1] In recent years, the clinical benefits of endovascular reperfusion therapy (ERT) for acute ischemic stroke (AIS) have emerged. The evidence was proven by the publication of five emergency thrombectomy randomized controlled trials (RCTs) from (2010- 2015) in Australia, Europe, and the United States of America, summarized by Tsang and colleagues here.[2] Nevertheless, LVOS still leads to critical neurological fatality and morbidity.[3] Furthermore, standard intravenous thrombolysis (IVT) is inefficient in attaining timely reperfusion, and the optimum medical therapy (MT) of victims with AIS remains questionable. [2,3]
Since the 1950s, numerous approaches have been attempted.[4] However, the application of catch-all terms VB insufficiency (VBI) or VB territory infarction covering all posterior circulation ischemia (PCI) events was observed.[4,5] Therefore, VBI can be described as transient ischemia of the VB circulation, most commonly characterized by dizziness, double vision, vertigo, loss of vision, headaches, vomit, ataxic manifestations, loss of balance, and bilateral body instability. [6]

Anatomically, the basilar artery (BA) comprises the union of two vertebral arteries (VA) while the posterior inferior cerebellar artery (PICA) and anterior spinal artery (ASA) are significant divisions of the VA, as the BA ascends, forming the anterior inferior cerebellar artery (AICA), the pontine branches, and the superior cerebellar artery (SCA) and finally the posterior cerebral arteries (PCAs). [7] 
Clinically, the hallmarks of VBS confirm patchy distribution rather than an explicit transverse configuration due to its rostro-caudal structure, which is often not explained by the individual lesion. It is evident as brainstem stroke, exhibiting preoccupation of the long tracts bilaterally combined with cranial nerve (CN) shortfalls. Hence, crossed $\mathrm{CN}$ and sensory-motor deficits are characteristic. Likewise, locked-in syndrome is customarily marked by quadriplegia and aphasia. Corticobulbar tract entanglement can manifest as a consequence of both sided anterior pontine lesions. [8]

Nearly $1 / 3(37 \%)$ cases of VBS are likely to be misdiagnosed in emergency settings, with over three times greater frequency than anterior circulation (AC) strokes.[9] Moreover, there is a marked deficiency of the extensive research on posterior circulation (PC) pathologies compared to the AC diseases.[10] VBS carries the risk of higher mortality compared to AC.[11] Likewise, the diagnosis of VBS in patients may manifest with dizziness or sudden onset of vertigo, as the symptoms mimic a mixture of common inner otologic complications, uncommon neurologic conditions, and mass lesions in the posterior fossa misdiagnosis can result in conflicting outcomes for the victims. [12] Indeed, the literature highlighted above exposes minimal extensive research on the PC region compared to the AC region and critical mortality of the LVOS in the posterior circulation, conflicting management consensus, and overwhelming VBS misdiagnosis.
This narrative review aimed to highlight the most recent global epidemiology of the VBS. Furthermore, this review will critically evaluate VBS management by analyzing progress treatment options, future directions and comparing and contrasting different treatment time windows and VBS prognosis determination based on contemporary literature.

\section{Method}

Electronic databases (PubMed, ScienceDirect, and Google Scholar) were searched for full papers and abstracts written in English. The following key terms or their combinations were used "vertebrobasilar stroke," "epidemiology," "Africa," "posterior circulation stroke," AND "prognosis." The search strategy yielded a total of 6,850 articles. This narrative review aimed to focus on the contemporary VBS perspectives; therefore, both full text and abstracts published between January 2016 and January 2020, regardless of study population or location, were analyzed and filtered. After duplicates were removed, 394 remained.

Based on title/abstract review, 202 articles were eliminated to focus on posterior circulation stroke. In comparison, seven article abstracts were not published in English, and 90 articles did not fulfill the inclusion criteria (epidemiology, management, or prognosis). After the screening process, 94 articles were incorporated into this review as shown in Figure 1.

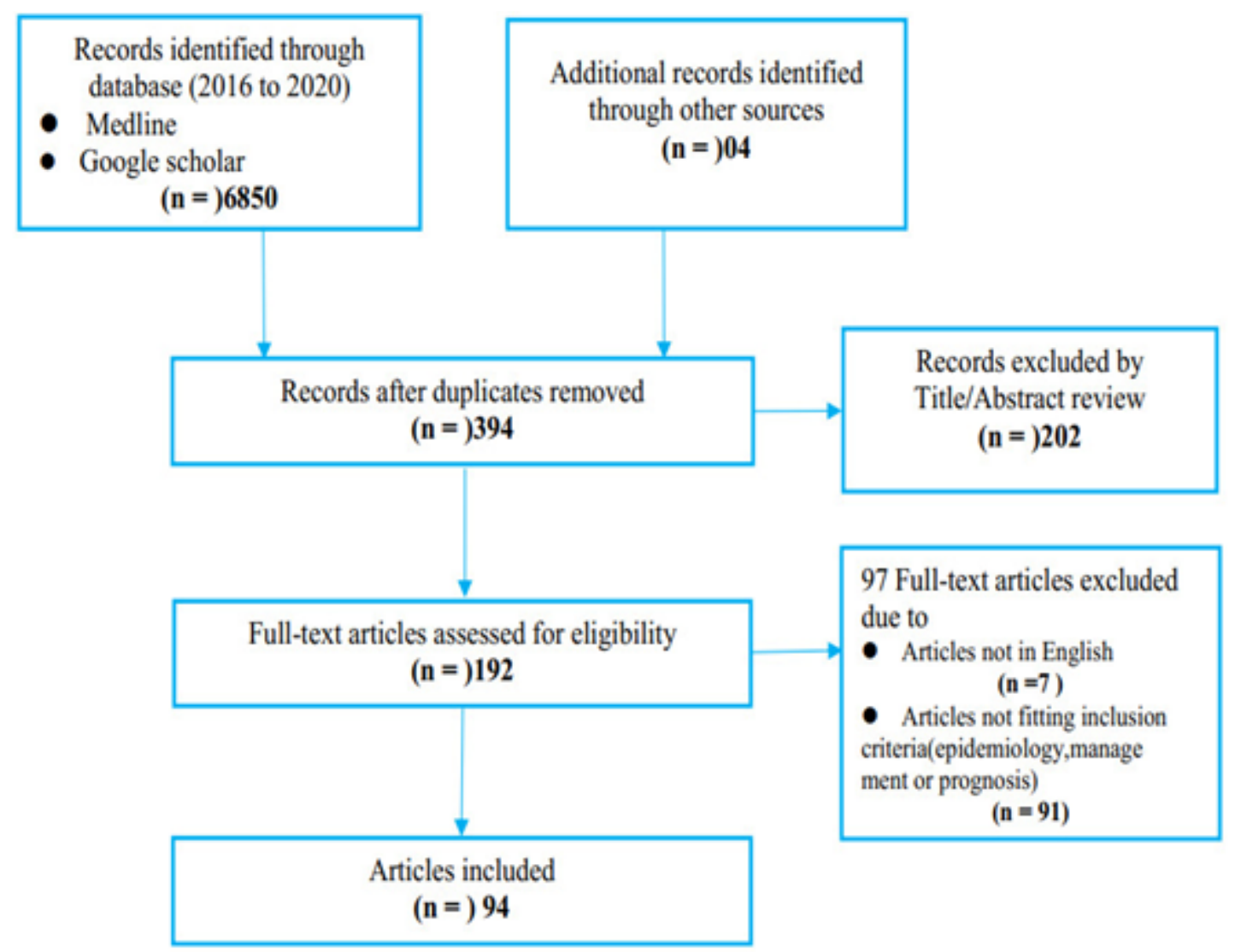

Figure 1. Flowchart for article selection 


\section{Epidemiology}

Globally, stroke is the 2nd principal cause of death and a dominant etiology of disability. [13] IS leads to a marked increase in morbidity and fatality, leading to high healthcare costs. Intracranial atherosclerotic disease is estimated to cause $10-15 \%$ of IS in Western nations, yet it is an underlying pathology in up to $54 \%$ of ISs in Asian communities.[14] In the Chinese population, it is currently the leading cause of mortality, most prevalently affecting men.[15,16]

VBS accounts for nearly $1 / 5$ of all ISs with acute BA occlusion linked to mortality and significant disabilities in more than $1 / 3$ of the victims. Unfortunately, its optimal therapy remains unestablished. Moreover, an estimated 20-24.8\% of these cases manifest with isolated vertigo from $1 / 6$ to $1 / 3$ cases, resulting in misdiagnosis as peripheralvestibular pathologies and undesirable rates of morbidity and mortality. Likewise, stroke and hypoperfusion prevalence are more prominent in victims with isolated vertigo and vascular risk factors, mainly incorporating hypoplasia, diabetes mellitus (DM), vertebral artery stenosis (VAS), and elevated vertebral artery curvature index (VACI).[17-19]

Intracranial LVOS (ILVO) is a dominant vascular pathology found in patients with stroke among Africans, Hispanics, and Asians. In contrast, extracranial LVOS is more common among Caucasians and the Middle Eastern (ME) population. In $\mathrm{ME}$ nations, $77 \%$ of strokes occur extracranially, with $11 \%$ occurring in VA, predominantly in men with an estimated mean age of 61-70.[20,21] Comparatively, in a West African study involving 1,191 participants, VBS with ischemic etiology accounted for $5.1 \%$ of all ISs, with $44.2 \%$ of the cases being $\geq 65$ years with an average age of 58 years, predominantly occurring in men residing in nonrural areas, and having hypertension was the most prevalent risk factor.[22]

In that context, these sex disparities in the prevalence and pattern of atheromatous plaque are explained by the anatomical study. Ogeng'o et al. found the length, diameter, conflux, and bifurcation angles exhibiting sexually dimorphic pattern, with the BA being notably wider for $4.5 \mathrm{~mm}$ and longer for mean length $27.1 \mathrm{~mm}$ in females contrasted to men.[23] Additionally, $79.8 \%$ of conflux occurred at the bulbopontine sulcus, comparable to the Indians but much higher than in the Polish and Turkish populations. Therefore, females may be more susceptible to atherosclerosis of the VB junction.
This should assist prospective designs and utilization in diagnostic and interventional schemes.[23] VBS account for $37 \%$ of $<2 \mathrm{~cm}$ cerebellar infarctions (CI) (small CI strokes), which frames $1 / 3$ of the ISs in the posterior location, which most commonly involve the posterior lobe and occasionally the anterior and flocculonodular lobes with the favorable clinical outcome as compared to large CIs.[24]

PCA territory is responsible for most embolic stroke of undetermined source, majorly subclinical atrial tachyarrhythmia. However, additional studies are required to review the efficacy of incorporating the infarction site in therapeutic choice-making and recognizing candidates for oral anticoagulation.[25] Based on the radiological conclusions in younger patients $\leq$ 50 years, the patent foramen ovale is considered a frequent source of cortical stroke.[26]

In the largest study, a meta-analysis of retrospective observational studies involving 3,875 acute IS cases, the prevalence of vertebral artery hypoplasia (VAH) was $18.6 \%$, predominantly in men, and had a significantly higher probability in PCI patients compared to patients with AC ischemia. However, further investigation in future prospective study protocols is warranted.[27] Additionally, PCI central stenosis and DM are also suggested as independent risk factors for PCI in patients with isolated vertigo.[28,29] Cases with PC infarction are at higher risk of reoccurring strokes with earlier onset, particularly in VB stenosis or hypoplasia.[30] Arterial calcification (AC) is more eminent in significant artery atherosclerosis. It accounts for the $42 \%$ of the IS risk factor in the VB arteries, with a marked association to age, $\mathrm{DM}$, and CAD.[31]

In the pediatric population, hospital-based VBS prevalence is estimated to be 8.1 per 100,000 children, while the crude mortality rate is 0.11 per 100,000 person-years in up to $71 \%$ cases presenting as impaired consciousness.[32] Furthermore, VAH heightens the risk of PCI in pediatric cases as an independent risk factor.[33]

VB stenosis occurs in an estimated $20 \%$ of VBS and poses tremendous danger for early stroke recurrence.[34] The proximal BA up to $24 \%$ and two-sided thalamic infarction is the most frequent occlusion section in subjects with intracranial atherosclerotic stenosis (IAS). In contrast, the distal portion is regularly observed in cases without IAS.[35] 
VB dolichoectasia (VBD) is marked by ectasia, elongated, and tortuous VBA. Pathophysiologically, it is linked to the degeneration of the inner elastic lamina, media thinning, and atrophic smooth muscle. Association with IS, thromboembolic events, autosomal disorders, and confined pathologies has been documented.[36] VBD prevalence ranges from $0.05-26.6 \%$ with male gender as the risk factor.[37,38] Likewise, there is no disparity in the prevalence of BA dolichoectasia between the rural and other populations. [39]

VB aneurysms are linked to poor natural history with the tremendous growth of 6 to $6.5 \%$ annually and rupture rates of $1.5-3 \%$ annually. Dolichoectatic aneurysm (DA) is the most prevalent form and is associated with more significant IS rates, followed by fusiform aneurysm (FA) and transitional aneurysm. By contrast, FA has a rupture hazard than DA.[40,41] Additionally, dolichoectasia imaging studies have proven the existence of a strong association between PCA territory and higher rates in the occurrence of old infarctions, and on the other side, acute lesions with the occurrence in the brain stem and white matter regions and rarely the cortico-subcortical area.[42]

The prognosis of fusiform VBD aneurysms is poor, with nearly $1 / 2$ of the cases worsening or progressing their aneurysm in 5 years. [43] Approximately $42 \%$ of these aneurysms occur at the BA trunk, although they may also rarely occur at the low-lying $\mathrm{BA}$ bifurcation, BA-SCA, BA-AICA, VB junction, and distal VA.[44] Hypertension in up to (67-72\%) and PC intracranial atherosclerosis leads to VBD and vertebrobasilar DA. Other risks include DM, hyperlipidemia, cigarette smoking, and coronary artery disease (CAD). [36,41,45,46]

Fabry disease is among the rare causes of early-onset VBS, obliging physicians to render prospective risk evaluation and family counseling and achieve suitable management plans to avert a recurrence.[47] Another rare pathology, the giant cell arteritis-related CI events, carries a considerable mortality rate of $28 \%$, occurs most commonly in the VB territory, and is frequently diagnosed in patients who have encountered novel ocular ischemic manifestations with an exhibition of low inflammatory markers.[48]

\section{Management of VBS}

\section{MT}

Urgent, aggressive, and time-dependent reperfusion of the brain undergoing ischemia is the gold standard and the first objective therapy for both the MT and ERT. Moreover, intensive MT is necessary for both medically managed and stented cases. $[34,49,50]$

MT has been confirmed to be superior and efficient with few unfavorable consequences over ERT for both intracranial VB (IVB) disease and extracranial VA (EVA) stenosis based on the Stenting and Aggressive Medical Management for Preventing Stroke in Intracranial Stenosis (SAMMPRIS), the Vertebral Artery Stenting Trial (VAST), and the Vitesse Stent Ischemic Therapy (VISSIT) trials. However, the vast ERT procedural complexities rate encountered in the SAMMPRIS was potentially avoidable with accurate patient choice and further technological advancement.[14,51] Intravenous (IV) tissue plasminogen activator (tPA) was proposed as the first reliable and efficient thrombolytic agent. The proposal was accompanied by the introduction of more novel thrombolytics, anticoagulants, and antiplatelets.[49]

Based on the IS symptom onset time (SOT) or patient last known well (LKW) time, the first bolus of $10 \%$ of the dose $(0.9 \mathrm{mg} / \mathrm{kg})$ of IV alteplase (IVA) is administered up to 60 seconds. The dosage should not exceed $90 \mathrm{mg}$. Currently, IVA is the gold standard for the chosen cases managed within 3-4.5 hours. Moreover, the aspirin regimen is commonly suspended until the following 24 hours but never substituted for the AIS approach in IV alteplase-/ ERT-eligible cases.[3,52] Revascularization for asymptomatic VA disease (VAD) is contraindicated regardless of the level of severity. Instead, aspirin (ASA), or clopidogrel if ASA is intolerable, and statins are suggested.[53] In contrast, the treatment of symptomatic VAS remains debatable, and there are no definite advantages or disadvantages for ERT when contrasted to MT only in the 4 RCTs involving 370 cases.[54]

Intra-arterial MET bears a higher recanalization rate compared to intra-arterial thrombolysis (IAT) in management of acute BA occlusion (ABAO). More significant probability of survival and a higher desirable clinical consequence at 90 days was documented. The ongoing 3-year, multicenter RCT, with forecasted 344 cases, evaluating ABAO within 8 hours of determining occlusion time, is presumed to clarify whether ERT plus standard MT is superior to standard MT solely for this PC region, 
the doubts which have already been resolved for the AC. $[55,56]$ By contrast, the proof of the preventive role of statin therapy on the progress of symptomatic BA stenosis and diminishing the risk of consequent AIS is verified. Nevertheless, large RCTs are required to validate this conclusion .[57]

Generally, in AIS management, acute BP lowering is contraindicated, except for cases in which BP is quite high $>185 / 110 \mathrm{mmHg}$ and IVA is expected. Moreover, the initial treatment of hypertension (HTN) in the first 24 hours by an initial decrease of $\mathrm{BP}$ by $15 \%$ is indicated in the evidence of critical acute comorbidities symbolizing endorgan damage. Cases with $\mathrm{BP} \geq 220 / 120 \mathrm{mmHg}$ without offered MET or ERT or the presence of comorbidities bears the same recommendation. Meanwhile, IV labetalol/nicardipine has been confirmed as an antihypertensive of choice. Further, BP should be maintained at $<180 / 105$ $\mathrm{mmHg}$ for at least the initial 24 hours after IVA. Otherwise, contemporary guidelines suggest letting the BP remain as high as 220/120 mmHg for maintaining the ischemic penumbra flow and limiting collateral flow breakdown. However, unnatural BP elevation in conventional stroke treatment is contraindicated.[1,3,58] Among a subgroup of VB disease and low flow, tight BP control $<140 / 90$ may raise the risk of consequent stroke.[59]

Additionally, added MT management includes antipyretics and exterior cooling for hyperthermia $\left(>38^{\circ} \mathrm{C}\right)$. Management of hyperglycemia to 7.8 $10.0 \mathrm{mmol} / \mathrm{L}$ blood glucose (BG) level, prevention of hypoglycemia (BG $<3.3 \mathrm{mmol} / \mathrm{L}$ ), blood cholesterol check, optimal antiplatelet treatments, and lifestyle adjustments are recommended. $[50,58,60]$

\section{Endovascular Treatment}

There has been a marked increase in ERT application in AIS management, and it is presently acknowledged as the gold standard procedure for the chosen cases. However, it necessitates the availability of proficient interventional neuroradiologists with adequate expertise. Furthermore, in the absence of contraindication, it should not point to delays in the initiation of the established IV thrombolysis (IVT) regimen. When implemented in tertiary stroke units with large capacity and skilled multidisciplinary stroke experts, significant results are achieved.[14,49,6163] Mechanical thrombectomy (MET) consists of thrombus retraction, aspiration technique, or a stent retriever device (SRD); however, SRD should prevail as the first choice. $[3,55]$
Additionally, IV alteplase ineligible victims can securely undergo ERT and appear to accomplish similar rates of reperfusion and high-grade result as MET plus ERT victims within the identical time frame of fewer than 6 hours.[64] There is emerging evidence of thoughtfully applying ERT (MET with SRD) for precisely selected VBS in-patients in whom groin puncture (GP) was started within 6 hours of SOT; however, the benefits are still doubtful. By contrast, several prospective studies on VBO have confirmed the possibility of considering intracranial stenting and multimodal recanalization for managing inpatients with medically uncontrollable strokein-evolution (SIE) 6 hours post-SOT. Nevertheless, the retrospective view of these studies, the small number of subjects, and the absence of a control group are the major weaknesses.[3,64-66]

In carefully chosen cases with AIS secondary to LVO in the PC presenting after 6 hours, ERT can be counted for patients with favorable multimodal imaging profiles considering the risks and advantages.[67] The most recent published single-center cohort study comprising 110 VBS cases proved that ERT is comparably safe and efficient, with low mortality rate and enhanced positive results as opposed to no therapy at all. The research recruited cases with the median National Institutes of Health Stroke Scale (NIHSS) of 31 and the median SOT to GP of 300 minutes. However, the study was confined by its retrospective essence, absence of a straight comparison of the various management modalities, lacking most contemporary stroke workflow, and application of old imaging techniques.[68]

Aggressive ERT should be offered following imaging victims with BAO with PC Alberta Stroke Program Early Computed Tomography Score (PCASPECTS) of $<8$ at baseline diffusion-weighted imaging (DWI).[69] Bowen et al. reported 935 LVOS cases who underwent ERT, and 13\% were VBS cases. Successful reperfusion was attained in $93 \%$ of the cases with mTICI score $2 b-3$, and $72 \%$ of them had good 3-month outcome, but $10 \%$ sustained mortality, confirming that ERT is secure and convenient for LVOS cases manifesting with mild NIHSS $\leq 8$. Yet, prospective controlled studies are required.[70] MET can be performed securely in VBS cases regardless of renal function. However, meticulous monitoring of these cases is needed following treatment. More comprehensive, prospective, multicenter studies assessing kidney dysfunction's impact on post-procedural intra-cerebral hemorrhage (ICH) are required.[71] 
Underlying IAS is prevalent in BAO and LVO cases. Thalamic infarction and pretreatment DWI are emerging predicting tools for IAS existence. Still, the operational time and ERT result for cases with and without IAS are comparable.[35,72] Although BAO has a significant mortality and morbidity rate, ERT of the VB tandem occlusions (VBTO) appears to be safe and achievable, with a high rate of favorable results. Additional studies are required to ascertain the safest technique to manage proximal stenosis.[73]

Although SAMMPRIS and VISSIT presumed ERT to be inferior to MT for cases with intracranial arterial stenosis, novel studies confirmed IVBS recurrence risk and mortality decrease and presence of similar consequences between MT and ERT 30 days post-procedure. Furthermore, contrasted to IVT or IAT, the MET has greater efficacy and safety in the management of acute VBO with notable reduction of NIHSS scores and excellent prognosis by modified Rankin Scale $(\mathrm{mRS}) \leq 2$ in $50.0 \%$ of the cases at 90 days. $[74,75]$

Intracranial stenting is life-saving therapy when conventional treatment fails. Stent retrievers reduce the clot burden before stenting. Additionally, without preceding IV fibrinolytic therapy, anti-platelet therapy can be securely employed with no added risk in symptomatic ICH cases . [76] Stenting in extracranial stenosis is secure, with lower complexity rates. However, stroke risk does not appear to be reduced by angioplasty with stenting, as the proximal atherosclerotic EVA stenosis (EVAS) is secure and attainable, with an excellent patency rate. Other trials are expected to prove the striking conclusions obtained in this research.[77,78] In symptomatic EVAS, revascularization can be used for lesions $\geq 50 \%$ in subjects with repetitive ischemic episodes despite optimal MT. [53]

Notwithstanding the emergence of quick and efficient aspiration thrombectomy devices, SRD should be the first-line management for ERT. They confer that the tremendous rates of recanalization in the existence or non-existence of a supplementary approach seemed satisfactory for BA occlusion therapy.[3,21,49,67,79,80] In contrast, in-stent restenosis is the main hurdle for VB stenting, which accounts for approximately $18 \%$ primarily in smaller stent diameter and predicted by alcohol use.[81] Despite notable IS complications, aspiration pneumonitis, pneumonia, and sepsis rate, stent angioplasty may be a reasonably safe alternative with outstanding clinical results for victims with technically promising lesions.[82]
Although VA aneurysm interventions bear a notable risk of mortality of up to $21 \%$ and periprocedural stroke risk (PSR) of up to $23 \%$, flow diversion is a reasonable and practical approach for non-saccular aneurysms of the PC, with favorable neurologic consequences in up to $83 \%$ of cases. Nonetheless, new studies have focused on recognizing risk factors and optimizing case choice.[83]

\section{Prognosis}

ABAO is an overwhelming, severe disease that occurs in an estimated $1 \%$ of all strokes. Recanalization is linked with a 1.5 -fold decrease of death risk or dependence, and a 2-fold decrease mortality risk. Moreover, SOT to management time of $<12$ hours correlated with a higher recanalization rate and a lower $\mathrm{ICH}$ rate. [84] In a recent retrospective case series involving 214 VBS cases, smoking, lower initial NHISS score, and prosperous reperfusion state was correlated with positive consequences post-ERT.[61] Nevertheless, in BAO cases following SRT, SOT to GP, especially when GP, after 6 hours from SOT, baseline core infarct, and rate of embolic infarction, may lead to reduced rates of a favorable result.[85] Furthermore, at a low dosage, tirofiban does not pose a risk of symptomatic ICH and mortality at 3 months in acute VBO cases following MET.[86]

Following SRD (Solitaire AB) thrombectomy, embolic stroke, and a higher value of pc-ASPECTS on DWI present favorable prognosis (Figure 2) of the acute PC strokes (PCS) due to ILVO. In the same way, suitable collateral status and low median baseline NIHSS was observed with a good prognosis. By contrast, in the retrospective research involving 62 cases with AIS due to BAO post-SRD thrombectomy, there was no variation in management consequences following ERT among IAS and non-IAS cases.[35,87]

Proper BAO long-term consequence prognostication controls sub-acute stage clinical therapy. NIHSS scale has been clinically confirmed to be a relevant prognostic tool in BAO cases at 24-48-hour. NIHSS thresholds of $\leq 4$ and $>22$ firmly prognosticate 30 -day high rates of positive and negative consequences, respectively.[88] Meanwhile, in isolated infarctions in a pontine region, BA disease is linked to a poor prognosis and progressive deficit (NIHSS $\geq 2$ scores in 5 days from SOT).[89] Furthermore, the expanded version of the NIHSS, the e-NHISS, appears to augment the sensitivity of the NIHSS in VBS evaluation with a positive impression on clinical trials as well due to added specific components in the current items of the NIHSS specifically for the exploration of symptoms and signs of the PCI as opposed 
to a limited NIHSS. However, additional studies involving a larger sample of patients are necessary to examine and establish the association between the e-NIHSS rate and neuroimaging data.[90]

On the other hand, the therapeutic influence of recombinant TPA (r-TPA) is time-dependent. In a large cohort study, including 71,010 IS subjects, 11,924 were VBS cases. VBS patients were observed to suffer pre- and intra-hospital delays, including rt-PA initiation lags as opposed to cases with AC stroke. Moreover, prospective pre-hospital research necessitated verifying whether the noted time of therapeutic delays have a direct influence on the consequence of PCS. [91] VBD aneurysm growth was best predicted by size (above $10 \mathrm{~mm}$ ) and morphological structure (transitional or fusiform). Moreover, presence of T1 hyperintensity MRI/MRA signal in the vessel wall and a daughter sac appears to play the same role.[40]
With novel ultrasound assessment of the VA, abnormalities of VB flow are a notable sign of mortality risk, independent of risks of stroke severity and age.[92] Angiographically, small cerebellar infarction bears a more commendatory prognosis than large ones at 90 days, and 65\% functional independence (mRS) below two scores was noted. Additionally, less supratentorial white matter lucencies weight correlated with a more favorable prognosis for significant CBI cases. Meanwhile, VBO and initial mRS scores were significantly linked to crossed cerebellar diaschisis prognosis.[24,93] BA stenosis most regularly manifests as a perforator stroke with a lower recurrence rate (RR); yet, discharged cases with suboptimal MT doubles the risk of recurrence. By contrast, optimum MT in embolic infarct cases was associated with excellent prognosis with no recurrence, while $\mathrm{RR}$ in watershed infarcts is nearly 57\%.[94]

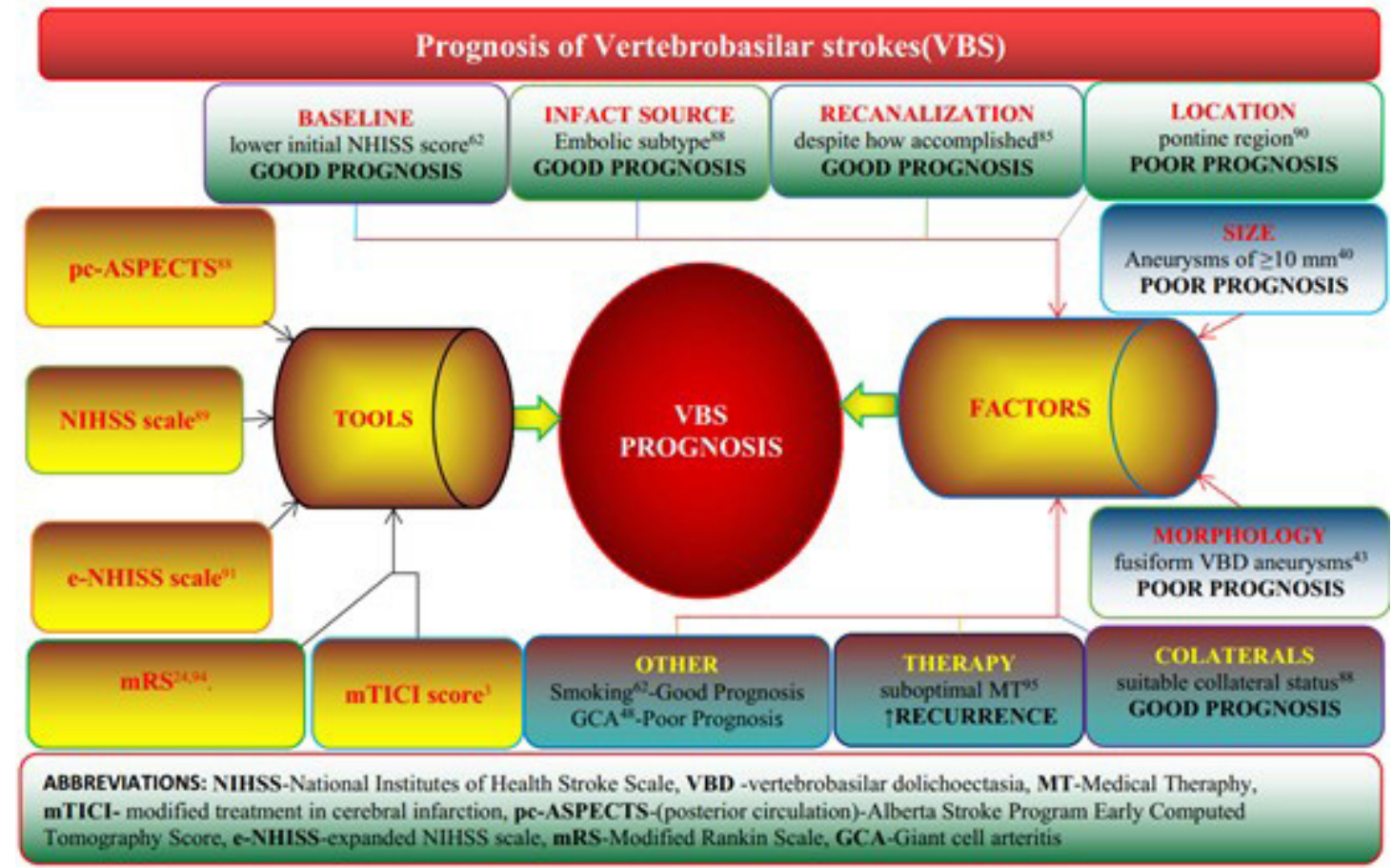

Figure 2. Prognostic factors, and tools used for Vertebrobasilar stroke prognosis determination (PubMed, ScienceDirect and Google Scholar, 2016 to January 2020) literature search.

\section{Limitations in the study design}

While appraising the studies summarized in this review, some limitations are considered due to the narrative nature of this review, which might predispose to bias. These include: (i) Search was narrowed to articles published in journals indexed in the central scientific databases, while articles published in conference proceedings were not incorporated.
In addition, this review consolidated all studies in the specified time frame highlighted in the methodology; no particular criteria were set to assess the quality of the articles. Furthermore, (ii)methodological similarities or differences and the number of participants were not considered during the assessment of the studies; (iii) strength of evidence, therapeutic protocols, or utilization of the conclusions were not set as inclusion/exclusion criteria. 


\section{Conclusion}

The epidemiology of VBS varies globally. ERT is increasingly utilized for VBS management. Prognostic factors and tools have been covered in detail in this review. However, prospective, multicenter, controlled studies comprising abundant patients are needed to clarify the time window dilemmas and whether ERT plus standard MT is superior to standard MT alone for this region.

\section{Contributions}

SSM contributed to this paper, with the literature search, designing, drafting, revising, editing and approval of the final manuscript.

\section{Funding}

There is no funding to disclose.

\section{Acknowledgements}

The author express thanks to Prof. Xianglin Cheng, Dr. Yan Li and Dr. Jian Zhao of Stroke Center and the Department of Neurology, The First Affiliated Hospital of Yangtze University for their expert inputs in revising this paper.

\section{Conflict of interest}

The author declare that no competing interest exists.

This article is published open access under the Creative Commons Attribution-NonCommercial NoDerivatives (CC BYNC-ND4.0). People can copy and redistribute the article only for noncommercial purposes and as long as they give appropriate credit to the authors. They cannot distribute any modified material obtained by remixing, transforming or building upon this article. See https:// creativecommons.org/licenses/by-nc-nd/4.0/

\section{References}

1. Brust JCM. Current diagnosis \& treatment neurology. McGraw Hill Professional; 2018.

2. Tsang ACO, Yeung RWL, Tse MMY, Lee R, Lui WM. Emergency thrombectomy for acute ischaemic stroke: Current evidence, international guidelines, and local clinical practice. Hong Kong Med J. 2018;24:73-80.

3. Powers WJ, Rabinstein AA, Ackerson T, Adeoye OM, Bambakidis NC, Becker K, et al. 2018 Guidelines for the Early Management of Patients With Acute Ischemic Stroke: A Guideline for Healthcare Professionals From the American Heart Association/ American Stroke Association. Stroke. Stroke; 2018;49:e46-110.
4. Caplan LR, Kim JS. Large vessel occlusive disease of the posterior circulation. Caplan's Stroke. 2016. p. 252-86.

5. Pirau, Letitia FL. Vertebrobasilar Insufficiency - StatPearls - NCBI Bookshelf. StatPearls [Internet]. Treasure Island (FL): StatPearls Publishing; 2019.

6. Neto ACL, Bittar R, Gattas GS, Bor-Seng-shu E, De Lima Oliveira M, Da Costa Monsanto $\mathrm{R}$, et al. Pathophysiology and diagnosis of vertebrobasilar insufficiency: A review of the literature. Int. Arch. Otorhinolaryngol. Georg Thieme Verlag; 2017. p. 302-7.

7. Lumley JSP, Craven J, Abrahams P, Tunstall R. Bailey \& Love's Essential Clinical Anatomy. CRC Press; 2018.

8. Robert B. Bradley Neurology in Clinical Practice 7e. Elsevier ;2016;

9. Tarnutzer AA, Lee SH, Robinson KA, Wang $Z$, Edlow JA, Newman-Toker DE. ED Misdiagnosis of cerebrovascular events in the era of modern neuroimaging. Lippincott Williams and Wilkins. Neurology ;2017. p. 1468-77.

10. Alexander MD, Rebhun JM, Hetts SW, Kim AS, Nelson J, Kim H, et al. Lesion location, stability, and pretreatment management: Factors affecting outcomes of endovascular treatment for vertebrobasilar atherosclerosis. J Neurointerv Surg. 2016;8:466-70.

11. Serles W, Gattringer T, Mutzenbach S, Seyfang L, Trenkler J, Killer-Oberpfalzer $\mathrm{M}$, et al. Endovascular stroke therapy in Austria: A nationwide 1-year experience. Eur J Neurol. 2016;23:906-11.

12. Edlow JA, Newman-Toker D. Using the Physical Examination to Diagnose Patients with Acute Dizziness and Vertigo. $J$ Emerg Med. 2016;50:617-28.

13. Katan M, Luft A. Global Burden of Stroke. Semin Neurol.; 2018;38:208-11.

14. Hussain M, Datta N, Cheng Z, Dornbos D, Bashir A, Sultan I, et al. Spanning from the West to East: An updated review on endovascular treatment of intracranial atherosclerotic disease. Aging Dis. 1;8(2) 2017. p. 196-202. doi: 10.14336/ AD.2016.0807. eCollection

15. Gao Y, Jiang B, Sun H, Ru X, Sun D, Wang $\mathrm{L}$, et al. The burden of stroke in China: Results from a nationwide population-based epidemiological survey. PLoS One; 2018;13. 
16. Qi Li, Hao Wu, Wei Yue, Qingqing Dai, Hui Liang, Hetao Bian, et al Prevalence of Stroke and Vascular Risk Factors in China: A Nationwide Community-based Study. Sci Rep.; 2017;7 Article number: 6402 .

17. Venhovens J, Meulstee J, Verhagen WIM. Acute vestibular syndrome: a critical review and diagnostic algorithm concerning the clinical differentiation of peripheral versus central aetiologies in the emergency department. J. Neurol. Dr. Dietrich Steinkopff Verlag GmbH and Co. KG; 2016. p. 2151-7.

18.Lattanzi S, Silvestrini M. Future challenges of stroke treatment. Neurol. Neurochir. Pol. Urban and Partner, 2018. p. 415-6.

19.Zhang DP, Li HR, Ma QK, Yin S, Peng YF, Zhang HL, et al. Prevalence of Stroke and Hypoperfusion in Patients With Isolated Vertigo and Vascular Risk Factors. Front Neurol. Frontiers Media S.A.; 2018;9.

20. Saber H, Amiri A, Thrift AG, Stranges S, Bavarsad Shahripour R, Farzadfard MT, et al. Epidemiology of Intracranial and Extracranial Large Artery Stenosis in a Population-Based Study of Stroke in the Middle East. Neuroepidemiology. S. Karger $A G ; 2017 ; 48: 188-92$.

21. Cohen JE, Leker RR, Gomori JM, Eichel $\mathrm{R}$, Rajz G, Moscovici S, et al. Emergent revascularization of acute tandem vertebrobasilar occlusions: Endovascular approaches and technical considerationsConfirming the role of vertebral artery ostium stenosis as a cause of vertebrobasilar stroke. J Clin Neurosci. Churchill Livingstone; 2016;34:70-6.

22. Dabilgou AA, Napon C, Drave A, Adeline Kyelem JM, Kabore J. Posterior circulation ischemic stroke in Burkina Faso. $J$ Neurol Stroke. 2018;8:235-8.

23. Ogeng'o JA, Mwachaka PM, Odhiambo DE, Sinkeet SR, Elbusaidy H, Olabu B, et al. Geometric features of vertebrobasilar arterial system in adult black Kenyans. Int $J$ Morphol. 2018;36:544-50.

24. Calic Z, Cappelen-Smith C, Cuganesan R, Anderson CS, Welgampola M, Cordato DJ. Frequency, Aetiology, and Outcome of Small Cerebellar Infarction. Cerebrovasc Dis Extra. S. Karger AG; 2017;7:173-80.

25. Makimoto H, Kurt M, Gliem M, Lee JI, Schmidt J, Müller P, et al. High incidence of atrial fibrillation after embolic stroke of undetermined source in posterior cerebral artery territory. $J$ Am Heart Assoc.; 2017;6 (12):e007448. doi: 10.1161/ JAHA.117.007448.
26. Stecco A, Quagliozzi M, Soligo E, Naldi A, Cassarà A, Coppo L, et al. Can neuroimaging differentiate $\mathrm{PFO}$ and AF-related cardioembolic stroke from the other embolic sources? Clinical-radiological correlation on a retrospective study. Radiol Medica. 2017;122:412-8.

27. Katsanos AH, Giannopoulos S. Increased risk for posterior circulation ischaemia in patients with vertebral artery hypoplasia: A systematic review and meta-analysis. Eur Stroke J. 2017;2:171-7.

28. Zhang DP, Lu GF, Zhang JW, Zhang SL, Ma QK, Yin S. Vertebral Artery Hypoplasia and Posterior Circulation Infarction in Patients with Isolated Vertigo with Stroke Risk Factors. J Stroke Cerebrovasc Dis. 2017;26:295-300.

29. Mitsumura H, Miyagawa S, Komatsu T, Hirai $\mathrm{T}$, Kono Y, Iguchi Y. Relationship between Vertebral Artery Hypoplasia and Posterior Circulation Ischemia. J Stroke Cerebrovasc Dis. 2016;25:266-9.

30. Chi HY, Hsu CF, Chen AC, Su CH, Hu $\mathrm{HH}, \mathrm{Fu}$ WM. Extracranial and intracranial ultrasonographic findings in posterior circulation infarction. J Ultrasound Med. 2018;37:1605-10.

31. Gökçal E, Niftaliyev E, Özdemir T, Kolukısa M, Asil T. The association of vertebrobasilar calcification with etiological subtypes, stroke recurrence and outcome in acute brainstem ischemic stroke. Neurol Neurochir Pol. 2018;52:188-93.

32. Carey S, Wrogemann J, Booth FA, Rafay MF. Epidemiology, Clinical Presentation, and Prognosis of Posterior Circulation Ischemic Stroke in Children. Pediatr Neurol. 2017;74:41-50.

33. Yang J, Shen Z, Wen H, Zhou H, Li C. The effect of vertebral artery hypoplasia in posterior circulation infarction in young patients. Int $J$ Neurosci. 2016;126:1092-6.

34. Drazyk AM, Markus HS. Recent advances in the management of symptomatic vertebral artery stenosis. Curr Opin Neurol. 2018;31:17.

35. Lee YY, Yoon W, Kim SK, Baek BH, Kim GS, Kim JT, et al. Acute basilar artery occlusion: Differences in characteristics and outcomes after endovascular therapy between patients with and without underlying severe atherosclerotic stenosis. Am J Neuroradiol. 2017;38:1600-4.

36. Samim M, Goldstein A, Schindler J, Johnson MH. Multimodality imaging of vertebrobasilar dolichoectasia: Clinical presentations and imaging spectrum. Radiographics. 2016;36:1129-46. 
37.ZdravkovićM, KostovM,PetrovicS,Zdravkovic J. Dolichoectasia of Vertebrobasilar Arteries as a Cause of Hydrocephalus and Ischemic Cerebral Stroke. Am J Forensic Med Pathol. 2017;38:359-61.

38.Peng YF, Zhang HL, Zhang DP, Zhao M, Zhang SL, Yin S. Perfusion by delayed time to peak in vertebrobasilar dolichoectasia patients with vertigo. Ann Clin Transl Neurol. 2018;5:1562-73.

39. Del Brutto OH, Mera RM, Del Brutto VJ, Costa AF, Zambrano M, Brorson J. Basilar Artery Dolichoectasia: Prevalence and Correlates With Markers of Cerebral Small Vessel Disease in Community-Dwelling Older Adults. $J$ Stroke Cerebrovasc Dis. 2017;26:2909-14.

40.Nasr DM, Brinjikji W, Rouchaud A, Kadirvel R, Flemming KD, Kallmes DF. Imaging characteristics of growing and ruptured vertebrobasilar non-saccular and dolichoectatic aneurysms. Stroke. 2016;47:106-12.

41.Nasr DM, Flemming KD, Lanzino G, Cloft HJ, Kallmes DF, Murad MH, et al. Natural History of Vertebrobasilar Dolichoectatic and Fusiform Aneurysms: A Systematic Review and Meta-Analysis. Cerebrovasc Dis. 2018;45:68-77.

42. Thijs V, Grittner U, Fazekas F, McCabe DJH, Giese AK, Kessler C, et al. Dolichoectasia and Small Vessel Disease in Young Patients with Transient Ischemic Attack and Stroke. Stroke. 2017;48:2361-7.

43.Xu DS, Levitt MR, Kalani MYS, RangelCastilla L, Mulholland CB, Abecassis IJ, et al. Dolichoectatic aneurysms of the vertebrobasilar system: Clinical and radiographic factors that predict poor outcomes. J Neurosurg. American Association of Neurological Surgeons; 2018;128:560-6.

44.Tjahjadi M, Niemelä M, Kivelev J, Serrone $\mathrm{J}$, Maekawa H, Jahromi BR, et al. Presigmoid Approach to Vertebrobasilar Artery Aneurysms: A Series of 31 Patients and Review of the Literature. World Neurosurg.2016;92:313-22.

45. Wang F, Hu XY, Wang T, Fang XM, Dai Z, Guo DL, et al. Clinical and imaging features of vertebrobasilar dolichoectasia combined with posterior circulation infarction: A retrospective case series study. Medicine (Baltimore). 2018;97 (48): e13166.

46. Matsukawa H, Shinoda M, Fujii M, Uemura A, Takahashi O, Niimi Y. Basilar dolichoectasia and the spontaneous intradural vertebral artery dissection. Brain Inj. 2016;30:90-4.
47. Majersik JJ. Inherited and Uncommon Causes of Stroke. Contin Lifelong Learn Neurol. 2017;23:211-37.

48. De Boysson H, Liozon E, Larivière D, Samson M, Parienti JJ, Boutemy J, et al. Giant cell arteritis-related stroke: A retrospective multicenter case-control study. J Rheumatol. 2017;44:297-303.

49. Asadi H, Williams D, Thornton J. Changing management of acute ischaemic stroke: The new treatments and emerging role of endovascular therapy. Curr Treat Options Neurol.; 2016;18.

50.Tsivgoulis G, Safouris A, Kim D-E, Alexandrov A V. Recent Advances in Primary and Secondary Prevention of Atherosclerotic Stroke. J Stroke. 2018;20:417-417.

51.Londo'o EML, Chaturvedi S. Stroke due to large vessel atherosclerosis Five new things. Neurol Clin Pract. 2016;6:252-8.

52.Allan H. Ropper, Martin A. Samuels, Joshua P. Klein SP. Adams and Victor's Principles of Neurology. Neurology. 2018.

53. Aboyans V, Ricco JB, Bartelink MLEL, Björck M, Brodmann M, Cohnert T, et al. 2017 ESC Guidelines on the Diagnosis and Treatment of Peripheral Arterial Diseases, in collaboration with the European Society for Vascular Surgery (ESVS). Eur. Heart J. 2018. p. 763-816.

54.Lattanzi S, Brigo F, Di Napoli M, Cagnetti C, Corradetti T, Silvestrini M. Endovascular treatment of symptomatic vertebral artery stenosis: A systematic review and metaanalysis. J Neurol Sci. 2018;391:48-53.

55.Liu X, Xu G, Liu Y, Zhu W, Ma M, Xiong $\mathrm{Y}$, et al. Acute basilar artery occlusion: Endovascular Interventions versus Standard Medical Treatment (BEST) Trial-Design and protocol for a randomized, controlled, multicenter study. Int J Stroke. 2017;12:77985.

56.56. Mak CHK, Ho JWK, Chan KY, Poon WS, Wong GKC. Intra-arterial revascularization therapy for basilar artery occlusion-a systematic review and analysis. Neurosurg Rev. 2016;39:575-80.

57. Yum KS, Chang JY, Jeong WJ, Lee S, Jeong $\mathrm{JH}$, Yeo MJ, et al. Effect of statin on progression of symptomatic basilar artery stenosis and subsequent ischemic stroke. PLoS One. 2017;12:1-10.

58. Wade S. Smith, S. Claiborne Johnston JCH. Cerebrovascular Diseases. Harrison's Princ Intern Med. 20e ed. New York: McGraw-Hill; 2018. p. 3068-94. 
59.Amin-Hanjani S, Turan TN, Du X, Pandey DK, Rose-Finnell L, Richardson DJ, et al. Higher Stroke Risk with Lower Blood Pressure in Hemodynamic Vertebrobasilar Disease: Analysis from the VERiTAS Study. $J$ Stroke Cerebrovasc Dis. 2017;26:403-10.

60. Roger P. Simon MJA. Clinical Neurology. 10e, editor. Stamford: McGraw Hill. 2018.

61. Bouslama M, Haussen DC, Aghaebrahim A, Grossberg JA, Walker G, Rangaraju $\mathrm{S}$, et al. Predictors of good outcome after endovascular therapy for vertebrobasilar occlusion stroke. Stroke. 2017;48:3252-7.

62. Mao Y, Nan G. Center volume and the outcomes of percutaneous transluminal angioplasty and stenting in patients with symptomatic intracranial vertebrobasilar stenoses: A meta-analysis. PLoS One. 2018;13:1-14.

63. Reyes S. P, Badilla O. L, Andreu D, Besa V, Rivera R, Sordo G, et al. Experiencia en el tratamiento endovascular del accidente cerebrovascular isquémico agudo en un centro chileno. Rev Med Chil. 2018;146:70816.

64. Rebello LC, Haussen DC, Grossberg JA, Belagaje S, Lima A, Anderson A, et al. Early endovascular treatment in intravenous tissue plasminogen activator-ineligible patients. Stroke. 2016;47:1131-4.

65. Seo WK, Oh K, Suh S Il, Seol HY. Intracranial stenting as a rescue therapy in patients with stroke-in-evolution. $J$ Stroke Cerebrovasc Dis.; 2016;25:1411-6.

66. He X, Zhang L, Yang J, Zheng H, Li K, Liu Y. Multimodal Therapy for Non-Superacute Vertebral Basilar Artery Occlusion. Interv Neurol. 2017;6:254-62.

67. Ko S-B, Park H-K, Kim BM, Heo JH, Rha J-H, Kwon SU, et al. 2019 Update of the Korean Clinical Practice Guidelines of Stroke for Endovascular Recanalization Therapy in Patients with Acute Ischemic Stroke. Neurointervention. 2019;14:71-81.

68. Rentzos A, Karlsson JE, Lundqvist C, Rosengren L, Hellström M, Wikholm G. Endovascular treatment of acute ischemic stroke in the posterior circulation. Interv Neuroradiol. 2018;24:405-11.

69. Guillaume M, Lapergue B, Gory B, Labreuche J, Consoli A, Mione G, et al. Rapid Successful Reperfusion of Basilar Artery Occlusion Strokes With Pretreatment Diffusion-Weighted Imaging PosteriorCirculation ASPECTS $<8$ Is Associated With Good Outcome. J Am Heart Assoc.; 2019;8.
70.Bowen MT, Rebello LC, Bouslama M, Haussen DC, Grossberg JA, Bianchi NA, et al. Clinical and Imaging Outcomes of Endovascular Therapy in Patients with Acute Large Vessel Occlusion Stroke and Mild Clinical Symptoms. Interv Neurol. 2018;7:91-8.

71.Laible M, Jenetzky E, Möhlenbruch MA, Neuberger U, Bendszus M, Ringleb PA, et al. Renal Impairment Is Associated with Intracerebral Hemorrhage after Mechanical Thrombectomy in Vertebrobasilar Stroke. Cerebrovasc Dis. 2019;47:48-56.

72. Baek J-H, Kim BM, Heo JH, Kim DJ, Nam HS, Kim YD. Endovascular and Clinical Outcomes of Vertebrobasilar Intracranial Atherosclerosis-Related Large Vessel Occlusion. Front Neurol. 2019;10:1-9.

73. Piechowiak EI, Kaesmacher J, Zibold F, Dobrocky T, Mosimann PJ, Jung S, et al. Endovascular treatment of tandem occlusions in vertebrobasilar stroke: Technical aspects and outcome compared with isolated basilar artery occlusion. $J$ Neurointerv Surg. 2020;12:25-9.

74. Ni GH, Liang C, Zhao WD, Tian XY, Sun HG, Gao SR, et al. Efficacy comparison of three vascular recanalization in patients with acute vertebrobasilar occlusion. Zhonghua Yi Xue Za Zhi. 2017;97:1001-5.

75. Abuzinadah AR, Alanazy $\mathrm{MH}$, Almekhlafi MA, Duan Y, Zhu H, Mazighi M, et al. Stroke recurrence rates among patients with symptomatic intracranial vertebrobasilar stenoses: Systematic review and metaanalysis. J Neurointerv Surg. 2016;8:112-6.

76. Delgado Acosta F, Jiménez Gómez E, Bravo Rey I, Bravo Rodríguez FA, Ochoa Sepúlveda JJ, Oteros Fernández R. Uso del stent intracraneal en el tratamiento endovascular en agudo del ictus. Radiologia. 2017;59:21825.

77. Markus HS, Larsson SC, Kuker W, Schulz UG, Ford I, Rothwell PM, et al. Stenting for symptomatic vertebral artery stenosis. Neurology. 2017;89:1229-36.

78. Che W, Jiang X, Dong H, Peng M, Zou Y, Song $\mathrm{L}$, et al. Effect of stenting for the proximal atherosclerotic extracranial vertebral artery stenosis. Chinese J Cardiol. 2017;45:34-8.

79. Wen WL, Li ZF, Zhang YW, Yang PF, Simfukwe K, Fang Y Bin, et al. Effect of Baseline Characteristics on the Outcome of Stent Retriever-Based Thrombectomy in Acute Basilar Artery Occlusions: A SingleCenter Experience and Pooled Data Analysis. World Neurosurg. 2017;104:1-8. 
80. Gory B, Eldesouky I, Sivan-Hoffmann R, Rabilloud M, Ong E, Riva R, et al. Outcomes of stent retriever thrombectomy in basilar artery occlusion: An observational study and systematic review. J Neurol Neurosurg Psychiatry. 2016;87:520-5.

81.Zheng D, Mingyue Z, Wei S, Min L, Wanhong C, Qiliang D, et al. The Incidence and Risk Factors of In-Stent Restenosis for Vertebrobasilar Artery Stenting. World Neurosurg.2018;110:e937-41.

82. Wajima D, Aketa S, Nakagawa I, Masui K, Yonezawa T, Enami T, et al. Effectiveness of Intracranial Percutaneous Transluminal Angioplasty or Stenting for Atherosclerotic Vertebrobasilar Artery Occlusion in the Acute Phase of Ischemic Stroke. World Neurosurg.; 2017;97:253-60.

83. Kiyofuji S, Graffeo CS, Perry A, Murad MH, Flemming KD, Lanzino G, et al. Metaanalysis of treatment outcomes of posterior circulation non-saccular aneurysms by flow diverters. J Neurointerv Surg. 2018;10:5006.

84. Chiang CC, Dumitrascu OM, Wingerchuk DM, O'Carroll CB. Acute Basilar Artery Occlusion: Does Recanalization Improve Clinical Outcome? A Critically Appraised Topic. Neurologist. 2018;23:71-4.

85. Phan K, Phan S, Huo YR, Jia F, Mortimer A. Outcomes of endovascular treatment of basilar artery occlusion in the stent retriever era: A systematic review and meta-analysis. $J$ Neurointerv Surg.2016;8:1107-15.

86. Quan T, Hou H, Xue W, Yu G, Ma H, Sun $\mathrm{J}$, et al. Endovascular treatment of acute intracranial vertebrobasilar artery occlusion: a multicenter retrospective observational study. Neuroradiology. 2019;61:1477-84.

87. Luo G, Mo D, Tong X, Liebeskind DS, Song L, Ma N, et al. Factors Associated with 90-Day Outcomes of Patients with Acute Posterior Circulation Stroke Treated By Mechanical Thrombectomy. World Neurosurg. 2018;109:e318-28.
88. Rangaraju S, Jovin TG, Frankel M, Schonewille WJ, Algra A, Kappelle LJ, et al. Neurologic Examination at 24 to 48 Hours Predicts Functional Outcomes in Basilar Artery Occlusion Stroke. Stroke. 2016;47:2534-40.

89. Gökçal E, Niftaliyev E, Baran G, Deniz Ç, Asil T. Progressive deficit in isolated pontine infarction: the association with etiological subtype, lesion topography and outcome. Acta Neurol Belg. 2017;1 17:649-54.

90. Olivato S, Nizzoli S, Cavazzuti M, Casoni F, Nichelli PF, Zini A. e-NIHSS: an Expanded National Institutes of Health Stroke Scale Weighted for Anterior and Posterior Circulation Strokes. J Stroke Cerebrovasc Dis. 2016;25:2953-7.

91. Sommer P, Seyfang L, Posekany A, Ferrari J, Lang W, Fertl E, et al. Prehospital and intrahospital time delays in posterior circulation stroke: results from the Austrian Stroke Unit Registry. J Neurol. 2017;264:131-8.

92. Muscari A, Bonfiglioli A, Magalotti D, Puddu GM, Zorzi V, Zoli M. Prognostic significance of carotid and vertebral ultrasound in ischemic stroke patients. Brain Behav. 2016;6:1-8.

93. Kang KM, Sohn CH, Choi SH, Jung $\mathrm{KH}$, Yoo RE, Yun TJ, et al. Detection of crossed cerebellar diaschisis in hyperacute ischemic stroke using arterial spin-labeled MR imaging. PLoS One. 2017;12:1-13.

94. Raghuram K, Durgam A, Kohlnhofer J, Singh A. Relationship between stroke recurrence, infarct pattern, and vascular distribution in patients with symptomatic intracranial stenosis. J Neurointerv Surg. 2018;10:11613. 